\title{
QUOTIENT FULL LINEAR RINGS
}

\author{
JOHN J. HUTCHINSON
}

\begin{abstract}
We define a ring $R$ to be an FL (full linear) ring if $R$ is isomorphic to the full ring of linear transformations of a left vector space over a division ring. $R$ is QFL if its left maximal quotient ring is an FL ring. In this paper we give necessary and sufficient conditions for a ring to be a QFL ring. We also generalize some results of Chase and Faith concerning subdirect sum decompositions of rings whose left maximal quotient ring is the direct product of FL rings.
\end{abstract}

If ${ }_{R} M$ is a left $R$-module, then $C\left({ }_{R} M\right)$ will denote the set of closed submodules of $M$. We say that $C\left({ }_{R} M\right)$ is atomic if $C\left({ }_{R} M\right)$ contains minimal nonzero elements, called atoms, and each nonzero element of $C\left({ }_{R} M\right)$ contains at least one atom. ${ }_{R} M$ is $Q$-prime if for any two atoms $I_{1}$ and $I_{2}$ of $C\left({ }_{R} M\right)$, there exists isomorphic submodules $X_{1}$ and $X_{2}$ of $M$ such that $X_{i}$ is essential in $I_{i}$ for $i=1$ and 2. If $X$ is a subset of $M$, then $l_{R}(X)$ will denote the left annihilator in $R$ of $X$.

THEOREM 1. $R$ is $Q F L$ if and only if the following conditions are satisfied:

(i) $Z\left({ }_{R} R\right)=0$,

(ii) $C\left({ }_{R} R\right)$ is atomic,

(iii) ${ }_{R} R$ is $Q$-prime.

Proof. Let $Q$ be the left maximal quotient ring of $R$. If conditions (i)-(iii) hold, then, by [2, p. 70], $C\left(_{Q} Q\right) \cong C\left({ }_{R} R\right)$ under contraction, and so $C\left({ }_{Q} Q\right)$ is atomic. Since $Z\left({ }_{R} R\right)=0$, we have that $Q$ is a left selfinjective regular ring. Thus $A$ is an atom of $C\left({ }_{Q} Q\right)$ if and only if $A$ is a minimal left ideal of $Q$. For the sake of simplicity of notation we will refer to the atoms of $C\left({ }_{Q} Q\right)$ and $C\left({ }_{R} R\right)$ as atoms of $Q$ and $R$ respectively.

Suppose $A_{1}$ and $A_{2}$ are atoms of $Q$. By [2, p. 70], $A_{1}$ and $A_{2}$ are injective as left $R$-modules. If $I_{1}=A_{1} \cap R$ and $I_{2}=A_{2} \cap R$, then $I_{1}$ and $I_{2}$ are atoms of $R$. Since ${ }_{R} R$ is $Q$-prime, there exists isomorphic left ideals $X_{1}$ and $X_{2}$ of $R$ such that $X_{i}$ is essential in $I_{i}$ for $i=1$ and 2. Clearly, $X_{i}$ is essential in $A_{i}$ for $i=1$ and 2 . The injectivity of $A_{2}$ gives that $A_{1}$ and $A_{2}$ are isomorphic. Thus $l_{R}\left(A_{1}\right)=l_{R}\left(A_{2}\right)$.

Received by the editors July 9, 1970.

AMS 1969 subject classifications. Primary 1650.

Key words and phrases. Maximal quotient ring, complete quotient ring, classical quotient ring, full linear ring, essential extension, subdirect sums.

Copyright (c) 1971, American Mathematical Society 
Let $S$ be the sum of the atoms of $Q$. If $\left\{A_{\alpha}\right\}$ is the set of atoms of $Q$ and $A \in\left\{A_{\alpha}\right\}$, then

$$
l_{R}(S)=\bigcap_{\alpha} l_{R}\left(A_{\alpha}\right)=l_{R}(A) .
$$

If $l_{R}(S) \neq 0$, then $l_{Q}(S) \neq 0$. By $\left[2\right.$, p. 71], $l_{Q}(S) \in C\left({ }_{Q} Q\right)$, and so there exists an atom $B$ of $Q$ such that $B \subseteq l_{Q}(S)$. But $B \subseteq S$, and so $B^{2}=0$. Since $B$ is idempotent generated we have a contradiction. Thus $l_{Q}(A) \cap R=l_{R}(A)=l_{R}(S)=0$, and so $l_{Q}(A)=0$. Since $A$ was an arbitrary atom of $Q$, we have that $l_{Q}(A)=0$ for all atoms of $Q$.

If $B$ is a nonzero left ideal of $Q$, then there is an atom $A$ of $Q$ such that $A \subseteq B$. Since $l_{Q}(B) \subseteq l_{Q}(A)=0$, we have that $Q$ is prime.

Let $W$ be an atom of $Q$. $W$ is a minimal left ideal of $Q$, and $W=Q e$ where $e^{2}=e \neq 0$. Since $Q$ is prime, we have that $V=e Q$ is a minimal right ideal of $Q$, and $D=e Q e$ is a division ring. Thus $V$ is a left $D$ vector space.

Let $L=\operatorname{Hom}_{D}(V, V)$, and define a map $q \rightarrow \bar{q}$ from $Q$ to $L$, where $x \bar{q}=x q$ for $x \in V$. This correspondence is clearly a ring homomorphism. If $q_{1}, q_{2} \in Q$ and $\bar{q}_{1}=\bar{q}_{2}$, then $x q_{1}=x q_{2}$ for all $x \in V$. Thus $e Q\left(q_{1}-q_{2}\right)=0$, and $q_{1}=q_{2}$ by the primeness of $Q$. Thus $q \rightarrow \bar{q}$ is a monomorphism of $Q$ into $L$.

Let $\bar{V}$ be the image of $V$ in $L$, and suppose $a \in L$ and $\bar{v} \in \bar{V}(v \in V)$. If $x \in V$, then $x=e x, v=e v, v a \in V$ and

$$
\begin{aligned}
x(\overline{v a}) & =x(v a)=(e x)(e v a)=(e x e)(v a) \\
& =(e x e v) a=(x v) a=(x \bar{v}) a=x(\bar{v} a) .
\end{aligned}
$$

Thus $\bar{v} a=\overline{v a} \in \bar{V}$, and so $\bar{V}$ is a right ideal of $L$. Since $\bar{V} \subseteq \bar{Q} \subseteq L$ we have that $L$ is a left quotient ring of $\bar{Q} \cdot \bar{Q}$ is left self-injective, so $\bar{Q}=L$. Thus $Q \cong L$, and so $R$ is $Q F L$.

Conversely, suppose $R$ is QFL. By $\left[2\right.$, p. 70], $Z\left({ }_{R} R\right)=0$ and $C\left({ }_{R} R\right) \cong C\left({ }_{Q} Q\right)$ under contraction. Thus $C\left({ }_{R} R\right)$ is atomic.

Suppose $I_{1}$ and $I_{2}$ are two atoms of $R$. Let $A_{1}$ and $A_{2}$ be atoms of $Q$ such that $A_{i} \cap R=I_{i}$ for $i=1$ and 2. Thus $A_{1}$ and $A_{2}$ are isomorphic under some isomorphism $f$, and $I_{i}$ is essential in $A_{i}$ for $i=1$ and 2. Let $X_{1}=f^{-1}\left(I_{2}\right) \cap I_{1}$ and $X_{2}=f\left(I_{1}\right) \cap I_{2}$. Clearly $X_{1} \cong X_{2}$ under the restriction of $f$ to $X_{1}$, and $X_{i}$ is essential in $I_{i}$ for $i=1$ and 2. Thus $R$ is $Q$-prime.

The following theorem is essentially a restatement of a theorem of Chase and Faith [1, Theorem 1.12].

THEOREM 2. Let $Q$ be the left maximal quotient ring of $R$. $Q$ is a direct product of $F L$ rings if and only if: 
(i) $Z\left({ }_{R} R\right)=0$,

(ii) $C\left({ }_{R} R\right)$ is atomic.

Proof. If (i) and (ii) hold, then $Z\left({ }_{Q} Q\right)=0, Q \cong \operatorname{Hom}_{R}(Q, Q)$, and $C\left({ }_{R} R\right) \cong C\left({ }_{Q} Q\right)$ under contraction. Thus $C\left({ }_{Q} Q\right)$ is atomic. By $[2$, p. 70], $C\left({ }_{Q} Q\right)$ consists of the direct summands of ${ }_{R} Q$, and so each direct summand of ${ }_{R} Q$ contains a minimal direct summand. By $[1$, Theorem 1.12], $Q$ is a direct product of FL rings.

Conversely, if $Q$ is a direct product of FL rings, then $Q$ is regular. Thus $Z\left({ }_{R} R\right)=0$, and $C\left({ }_{Q} Q\right)$ consists of the direct summands of ${ }_{R} Q$. Thus $C\left({ }_{Q} Q\right)$ is atomic, and since $C\left({ }_{R} R\right) \cong C\left({ }_{Q} Q\right)$ under contraction we have that $C\left({ }_{R} R\right)$ is atomic.

If $R$ is a subdirect sum of rings $\left\{R_{\alpha} \mid \alpha \in A\right\}$ and $S=\prod_{\alpha} R_{\alpha}$, then the subdirect sum is essential if $R$ (identifying $R$ and its canonical isomorphic image in $S$ ) is an essential left $R$-submodule of $S$. Some elementary properties of essential subdirect sums appear in [3].

THEOREM 3. Let $Q$ be the left maximal quotient ring of a ring $R$. $Q$ is the direct product of FL rings if and only if $R$ is an essential subdirect sum of $Q F L$ rings.

Proof. Suppose $Q=\prod_{\alpha} Q_{\alpha}$, where each $Q_{\alpha}$ is an FL ring with identity $e_{\alpha}$. For each $\alpha \in A$, let $R_{\alpha}=R e_{\alpha}$. Suppose $\alpha \in A$ and $0 \neq x_{\alpha} \in Q_{\alpha}$. Since $x_{\alpha} \in Q$ there is an $r \in R$ such that $0 \neq r x_{\alpha} \in R$. If $r_{\alpha}=r e_{\alpha}$, then

$$
0 \neq r x_{\alpha}=r e_{\alpha} x_{\alpha}=r_{\alpha} x_{\alpha} \in R_{\alpha} x_{\alpha} \cap R_{\alpha} .
$$

Thus each $Q_{\alpha}$ is a left quotient ring of the corresponding $R_{\alpha}$. Since $Q_{\alpha}$ is regular, we have that $Z\left({ }_{R_{\alpha}} R_{\alpha}\right)=0$, and $Q_{\alpha}$ is the left maximal quotient ring of $R_{\alpha}$. Thus each $R_{\alpha}$ is a QFL ring.

Clearly $R$ is a subdirect sum of the rings $R_{\alpha}\left(r \rightarrow\left\{r e_{\alpha}\right\}\right)$. Since $R \subseteq \prod_{\alpha} R_{\alpha} \subseteq Q$, we have that the subdirect sum is essential.

Conversely suppose $R$ is an essential subdirect sum of QFL rings $\left\{R_{\alpha} \mid \alpha \in A\right\}$. Let $Q_{\alpha}$ be the left maximal quotient ring of $R_{\alpha}$ and let $S=\prod_{\alpha} Q_{\alpha}$. Clearly $R \subseteq \prod_{\alpha} R_{\alpha} \subseteq S$.

If $x$ is a nonzero element of $S$, then for some $\alpha \in A$ we have 0 $\neq e_{\alpha} x \in Q_{\alpha}$, where $e_{\alpha}$ is the identity of $Q_{\alpha}$. Since $Q_{\alpha}$ is a left quotient ring of $R_{\alpha}$ it follows that

$$
0 \neq R_{\alpha} e_{\alpha} x \cap R_{\alpha}=R_{\alpha} x \cap R_{\alpha} .
$$

Since $R_{\alpha} x \cap R_{\alpha}$ is a nonzero $R$-submodule of $\prod_{\alpha} R_{\alpha}$, we have that $R_{\alpha} x \cap R_{\alpha} \cap R \neq 0$. Since $R_{\alpha} \cap R$ is an essential $R_{\alpha}$ (and $R$ ) submodule of $R_{\alpha}$, and since $Z\left({ }_{R_{\alpha}} R_{\alpha}\right)=0$, we have

$$
\left(R_{\alpha} \cap R\right)\left(R_{\alpha} x \cap R_{\alpha} \cap R\right) \neq 0 .
$$


Thus

$$
0 \neq\left(R_{\alpha} \cap R\right)\left(R_{\alpha} x \cap R_{\alpha} \cap R\right) \subseteq R x \cap R,
$$

and so $S$ is a left quotient ring of $R$. Since $S$ is regular, we have $Z\left({ }_{R} R\right)=0$. Thus $S=Q$, and $Q$ is the direct product of FL rings. Note that $R_{\alpha}=R_{\alpha} e_{\alpha}=r e_{\alpha}$, and so the QFL components of $R$ are uniquely determined.

\section{REFERENCES}

1. S. U. Chase and C. Faith, Quotient rings and direct products of full linear rings, Math. Z. 88 (1965), 250-264. MR 31 \#2281.

2. C. Faith, Lectures on injective modules and quotient rings, Lecture Notes in Math., no. 49, Springer-Verlag, New York, 1967. MR 37 \#2791.

3. J. Hutchinson, Essential subdirect sums of rings (to appear).

Washington State University, Pullman, Washington 99163 